\title{
A Neural Rumor Detection Framework by Incorporating Uncertainty Attention on Social Media Texts
}

\author{
Yan $\mathrm{Gao}^{1}, \mathrm{Xu} \mathrm{Han}^{2}$, and Binyang $\mathrm{Li}^{1(\bowtie)}$ \\ ${ }^{1}$ School of Information Science and Technology, \\ University of International Relations, Beijing, China \\ \{ygao,byli\}@uir.edu.cn \\ ${ }^{2}$ College of Information Engineering, Capital Normal University, \\ Beijing, China \\ hanxu@cnu.edu.cn
}

\begin{abstract}
Automatic rumor detection technology has become a very urgent need, as rumors can arise and spread dauntingly fast in social media, which bring unforeseeable and devastating impacts. However, current approaches mainly capture the event semantics or user-based features for rumor detection, but neglect the uncertainty expressions that strongly indicate the unverified nature of a rumor. As a result, these methods perform suboptimal when the topics of being verified rumors are changing wildly. In this paper, we present a neural rumor detection framework, namely NERUD. In NERUD, both uncertainty semantics and the event semantics of a word are represented by the attention mechanisms to generate a rumor representation for rumor detection. Experiments were conducted on the benchmark dataset and the Chinese Rumor Corpus (CRC), and the results showed that our NERUD outperformed state-ofthe-art approaches on CRC dataset, and the uncertainty semantics was proven effective on rumor detection task.
\end{abstract}

Keywords: Rumor detection $\cdot$ Deep learning $\cdot$ Neural network $\cdot$ Attention

\section{Introduction}

The concept of rumor has a long history, and there are various definitions from different research communities [1]. In this paper, we follow a widely accepted definition of rumor, that is, "an unverified statement towards an object, event, or issue of public concern, circulating from person to person with a context of uncertainty. This unverified statement is circulating without known authority for its truthfulness at the current time, but may turn out to be true, or partly or entirely false; alternatively, it may also remain unresolved" [16]. For simplicity, this paper will use "rumor" to refer to "false rumor", i.e., rumor that eventually is verified to be false. Since the false rumors are often related to events of public concern that are widely circulated among the public, the widespread of rumors will always cause public disturbed and threat social stability. 
Figure 1 illustrates two rumors generated from Sina Weibo and Twitter. As to Rumor 1, it was towards the earthquake occurred in Japan in 2011 that finally resulted in the nuclear crisis in Fukushima. This incident arouse the public anxiety and uncertainty about the nuclear radiation, and rumors were posted in both Twitter and Chinese Microblog platforms, which is spiking the demand in the U.S. and China for a cheap drug that can protect against the radiation damage, and leading to huge society chaos. Similarly, Rumor 2 was posted in Twitter in 2013 which caused severe social panic, and lead to great economic damage in the stock market.

\section{Rumor 1: 食用含碘盐很可能预防辐射。 \\ Salt could probably prevent people from nuclear radiation. \\ Rumor 2: Someone witnessed that Barack Obama was injured in an explosion in the White House.}

Fig. 1. Two examples of rumors. (Those words in bold express uncertain semantics, while those words underlined express the semantics of rumor-related events.)

So, there is an urgent need to automatically identify rumors in social media, so that the diffusion of harmful rumors can be stopped to minimize the negative impact.

To address this problem, existing research on rumor detection mainly used a wide variety of features incorporated into machine learning algorithms to identify rumors [12]. These features involve tweets statistics [13], topic-based features [15], user-based features [6], and location-based features [15], etc. Furthermore, propagation information was also taken into consideration $[4,9,14]$. More recently, some studies employ deep learning approaches into rumor detection by integrating the semantics representation of the topic or event $[7,8,11]$.

However, from the rumor definition, two aspect information should be accounted for rumor detection: (1) the representation of event of public concern; and (2) the context of uncertainty. The above approaches mainly focused on the information of event semantics, but ignore the uncertainty expressions that strongly indicate the unverified nature of a rumor. In fact, when producing a rumor, users often use uncertain descriptive information, such as it is said that..., likely, and etc. In Fig. 1, both rumors use uncertainty expressions, e.g. probably and witnessed. Different from event semantics that mainly represented by topical words, the uncertainty semantics are expressed more consistent, even if the topics of being verified events are changing wildly and frequently. So, we argue that current approaches only accounting for event semantics perform suboptimal for rumor detection in social media.

In this paper, we present a neural rumor detection framework, namely NERUD. In NERUD, both the event semantics and uncertainty semantics are represented by attention mechanisms to generate a rumor representation that facilitate the NERUD to identify rumors. Particularly, to the best of our knowledge, we are the first to exploits uncertainty semantics for rumor detection in social media texts. Furthermore, various sentence encoders are used in our model, including RNN, LSTM, and Bi-GRU, which 
are proven useful in text representation. Experiments were conducted on the benchmark dataset provided in [8] and the Chinese Rumor Corpus (CRC) constructed by ourselves, and the results showed that our NERUD outperformed state-of-the-art approaches on CRC dataset, and the uncertainty semantics was proven effective on rumor detection task. In addition, we also tested conventional machine learning models with and without uncertainty semantics, and the results confirmed that uncertain semantics could boost rumor detection of conventional models.

\section{Neural Rumor Detection Framework}

In this section, we will describe our neural rumor detection framework, namely NERUD. NERUD is consructed by twto parts: (1) Rumor detector, and (2) Uncertainty identifier. The rumor detector attempts to detect the rumor from the posts, while the uncertainty identifier aims to detect uncertainty expressions, which will be further integrated into rumor representation. The overall framework is illustrated in Fig. 2.

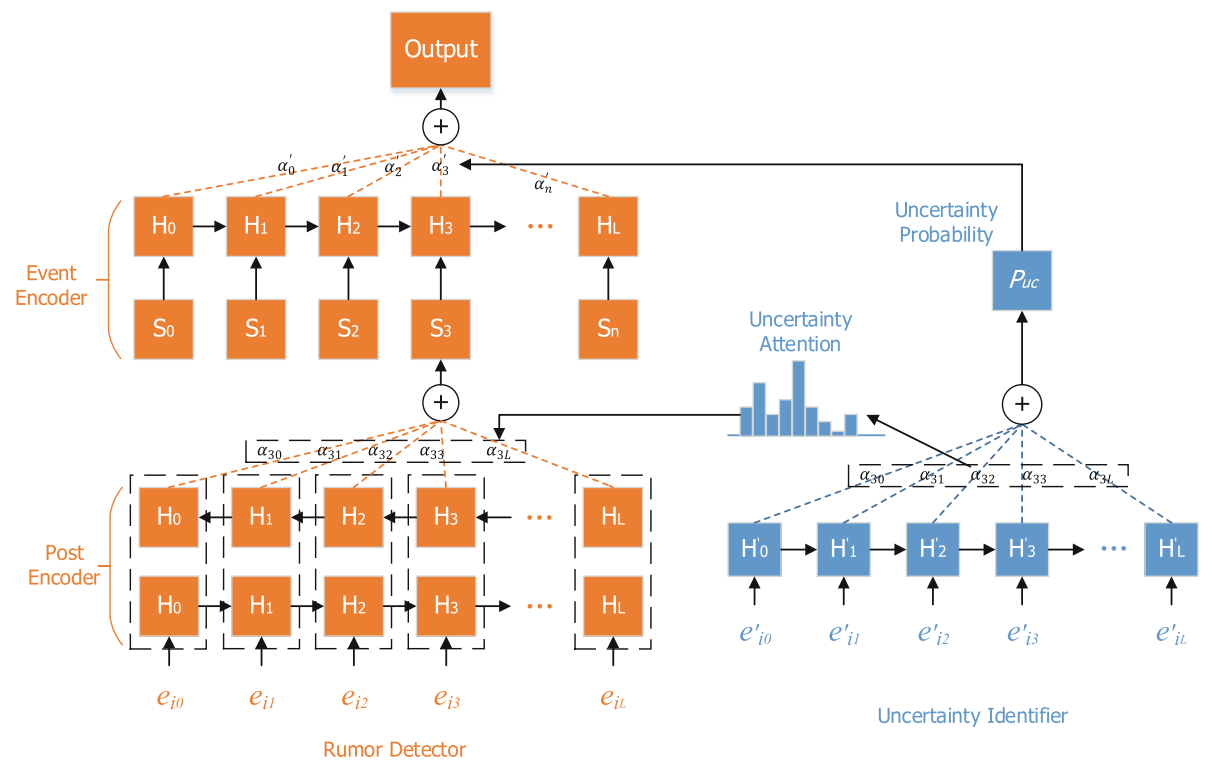

Fig. 2. The neural rumor detection framework by integrating uncertainty attention.

\subsection{Rumor Detector}

Without the loss of generality, an event can be defined as $E$, which consists of all the related posts $P_{i} \in E, i \in[0, T]$. A post can be formulated as a word sequence $P_{i}=\left\{x_{i j}\right\}, j \in[0, L]$ where $L$ denotes the length of $P_{i}$. The objective of rumor detection is produce y for each $E$, and $y \in\{$ rumor, non_rumor $\}$, that is to identify if the event is a rumor. 
More specifically, our rumor detector consists of 3 layers, i.e., a word embedding layer, a post encoder layer, and an event encoder layer.

In the word embedding layer, the pre-trained word vectors are used to transform each word $x_{i j}$ into a vector, and then all the word vectors are connected together to generate a tensor of word embeddings vector $e_{i j}$. In addition, all sentences are segmented into words with the stop words being removed, and we set the words out of vocabulary as zero-vectors.

Then, we will encode each single post and the corresponding event, respectively. We will firstly use bidirectional GRU network to encode the post. Because the rumourous content is often mixed in the normal texts, the position where the rumorous content exactly starting cannot be determined. One-layer GRU network cannot capture all the information of the rumorous texts. So we use bidirectional GRU, Bi-GRU [2] network as a basic model to learn the features of rumors. After that, we will encode the event based on the corresponding posts by using GRU network.

Post encoder: a bidirectional GRU network [2] is applied to obtain post information. We follow the classic bidirectional GRU settings and obtain the concatenation of the utterance embedding $h_{i j}=\left[\vec{h}_{i j}, \overleftarrow{h}_{i j}\right]$. Thus the final utterance of a post $\mathrm{h}_{\mathrm{ij}}$ can be calculated by:

$$
h_{i 0}: h_{i j}=\operatorname{BiGRU}\left(e_{i 0} \ldots e_{i j}\right) j \in[0, L]
$$

where $e_{i j}$ is the vectorised representation of each word. In the post encoder layer, the attention mechanism is used to find the words that are important to the post, the attention distribution $\alpha_{i j}$ represents the importance of $j^{\text {th }}$ word in the $i^{\text {th }}$ post:

$$
\begin{gathered}
\alpha_{i j}=\frac{\exp \left(\tanh \left(W_{w} h_{i j}+b_{w}\right)\right)}{\sum_{j} \exp \left(\tanh \left(W_{w} h_{i j}+b_{w}\right)\right)} \\
s_{i}=\sum_{j=0}^{L} \alpha_{i j} h_{i j}
\end{gathered}
$$

where $s_{i}$ is the embedding of $i^{\text {th }}$ post that summarizes all the information of each post.

Event encoder: To encode the event, all the posts belonging to the same event are all used. Given an event $E$, each post $P_{i} \in E$ is represented by a vector $s_{i}$, and the event utterance $h=\left\{h_{i}: h_{T}\right\}$ can be computed by:

$$
h_{i}: h_{T}=G R U\left(s_{0} \ldots s_{i}\right) i \in[0, T]
$$

Through the GRU encoder network, the event semantics can be represented by the attention vector $\alpha_{i}$ that measures the importance of each post vector.

$$
\begin{gathered}
\alpha_{i}=\frac{\exp \left(\tanh \left(W_{s} h_{i}+b_{s}\right)\right)}{\sum_{i} \exp \left(\tanh \left(W_{s} h_{i}+b_{s}\right)\right)} \\
d=\sum_{i=0}^{T} \alpha_{i} h_{i}
\end{gathered}
$$


where $d$ is the representation of an event. Then, a softmax function is used for rumor classification, and the training loss is calculated by cross entropy.

$$
p_{r}=\operatorname{softmax}(W d+b)
$$

In this way, we can obtain the event semantics representation, which will be further used in rumor detection. In order to the uncertainty expression, NERUD also incorporates uncertainty semantics into rumor detector, and well introduce in the following subsection.

\subsection{Uncertainty Identifier}

In order to represent the uncertainty semantics, we follow the work of [5], and integrate attention mechanism into sentence encoders for uncertainty detection. The purpose of this model is to learn the representation of uncertainty expression. To do this, we feed the vectorized representation of each word $\mathrm{w}_{\mathrm{i}}$ in a sentence sequentially to an encoder network, and produce the post encoder hidden state $h_{i j}^{\prime}$ by RNN-based encoders. During this procedure, the attention distribution $\alpha_{i j}^{\prime}$ is calculated by the following equations.

$$
\alpha_{i j}^{\prime}=\frac{\exp \left(\tanh \left(W_{h} h_{i j}^{\prime}+b_{h}\right)\right)}{\sum_{L} \exp \left(\tanh \left(W_{h} h_{i j}^{\prime}+b_{h}\right)\right)}
$$

The uncertainty attention $\alpha_{i j}^{\prime}$ indicates the importance of each word in uncertainty identifier. As a result, more attentions are paid to the words with uncertainty semantics, assigned with a higher weight. Then, the attention distribution will be used to generate the weighted sum of the hidden states of the encoder $h_{i}^{\prime}$.

$$
h_{i}^{\prime}=\sum_{j=0}^{L} \alpha_{i j}^{\prime} h_{i j}^{\prime}
$$

where $h_{i}^{\prime}$ is a vectorized representation of a post, which is fed through a softmax function to produce the uncertainty possibility distribution, and further to detect if the post is a rumor.

$$
p_{u i}=\operatorname{softmax}\left(W_{u} h_{i}^{\prime}+b_{u}\right)
$$

In this paper, RNN-based encoders have four variants, namely, RNN, LSTM, and Bi-GRU. Particularly, as Bi-GRU has two opposite directions, its uncertainty representation takes the concatenation of the last states from both directions, which come from two ends of a given context.

\subsection{Rumor Detection by Exploiting Uncertainty Semantics}

By now, we have acquired the semantics representation by using the uncertainty identifier. We will then employ the uncertainty attention into rumor detector. Rather than output the label of uncertainty identifier module, we employ the uncertainty 
distribution $\alpha_{i j}^{\prime}$ and the uncertainty representation $h_{i}^{\prime}$ into rumor detector. In NERUD, we integrate the uncertainty semantics into both post encoder layer and event encoder layer.

First of all, we will obtain the post representation with uncertainty attention by the following equations:

$$
\begin{gathered}
\alpha_{s_{i j}}=\frac{\exp \left(f\left(\alpha_{i j}^{\prime}, \alpha_{i j}\right)\right)}{\sum_{j=1}^{L} \exp \left(f\left(\alpha_{i j}^{\prime}, \alpha_{i j}\right)\right)} \\
f\left(\alpha_{i j}^{\prime}, \alpha_{i j}\right)=\tanh \left(W_{U} \alpha_{i j}^{\prime}+W_{R} \alpha_{i j}+b_{s}\right)
\end{gathered}
$$

where $f()$ is used to calculate the important words for representing the sentence, while $W_{U}, W_{R}$ and $b_{s}$ are the weights.

Therefore, in rumor detector, we use the attention distribution $\alpha_{s_{i j}}$ that incorporating uncertainty attention to replace the $\alpha_{i j}$ in Eq. (3). The final post representation $s_{i}$ can be expressed with the attention weight as $\alpha_{s_{i j}}$.

For the event encoder layer, the uncertainty probability distribution $p_{u i}$ is used to combine the event representation and the uncertainty representation to form a rumor representation as follows:

$$
\begin{aligned}
\alpha_{d_{i}} & =\frac{\exp \left(f\left(s_{i}, p_{u i}\right)\right)}{\sum_{i=1}^{T} \exp \left(f\left(s_{i}, p_{u i}\right)\right)} \\
f\left(s_{i}, p_{u i}\right) & =\tanh \left(W_{S} s_{i}+W_{P} p_{u i}+b_{d}\right)
\end{aligned}
$$

Similar with the post layer encoder, $\alpha_{i}$ in Eq. (5) is replaced by $\alpha_{d_{i}}$ which represent the combination of the event and the uncertainty semantics. Finally, the rumor representation with both event semantics and uncertainty semantics is used for rumor detection.

\section{Experiment}

In this section, we design several experiments to evaluate the performance of our model. We will firstly introduce the experiment setup, including the dataset, the approaches for comparison, and parameter tuning. Then we will evaluate the effectiveness of NERUD, and discuss the experimental results in details.

\subsection{Experiment Setup}

Our experiments are conducted on three datasets. The first one was created by [8] and widely used by many studies on Chinese rumor detection tasks. The second one was constructed by ourselves, namely Chinese Rumor Corpus (CRC). CRC contains 2,542 rumors that are officially reported by Sina community management center. We also collected the same number of non-rumors from Sina Weibo to construct the corpus. 
The details of the two datasets are shown in Table 1. Since we need to train our uncertainty identifier, we also use the Chinese benchmark dataset for uncertainty identification provided by [5].

In our experiment, precision, recall and F1-measure were used as the effectiveness evaluation metrics.

We utilized word2vec [10] to train Chinese word embeddings based on the experimental datasets. We set the dimensionality of the word vector as 150 , and the window length of max pooling as the maximum word length. To build up the vocabulary, we retain the word appearing more than three times and the word not in the vocabulary will be replaced with an unknown token.

Table 1. Statistics on two experimental datasets.

\begin{tabular}{l|l|l}
\hline Statistic & Ma's dataset & CRC dataset \\
\hline Posts & $3,805,656$ & $4,017,365$ \\
\hline Rumors & 2,313 & 2,542 \\
\hline Non-Rumors & 2,351 & 2,542 \\
\hline
\end{tabular}

During the training of the uncertainty attention model, we set the dimension of hidden size as 500 for RNN and LSTM. For the Bi-GRU encoder, which has two directions, we set the state size as 250 for each direction. We following the work by [3] with learning rate 0.01 for training. Since several sentence encoders are utilized in uncertainty attention module, we will firstly compare the performance of different sentence encoders on uncertainty identification task based on the benchmark dataset provided by [5].

From Table 2, we can see that the uncertainty identifier with Bi-GRU encoder achieves the best performance, so we will use Bi-GRU encoder in the following experiments.

For the rumor detector model, we use a Bi-LSTM network with 250 hidden states for each direction. The learning rate is set as 0.001 and the dropout rate is set 0.5 , both empirically. We only utilize $10 \%$ of Ma's dataset for parameter tuning, and the rest of the dataset were split with a ratio of 3:1 for training and test. Note that since NERUD exploits the uncertainty attention that will perform consistency based on different events, we also follow the same parameter setting for testing our CRC dataset.

Table 2. Comparison between different encoders.

\begin{tabular}{l|l|l|l}
\hline Encoders & Precision & Recall & F1 \\
\hline RNN & 0.712 & 0.680 & 0.696 \\
\hline LSTM & 0.778 & 0.785 & 0.781 \\
\hline Bi-GRU & $\mathbf{0 . 7 9 2}$ & $\mathbf{0 . 7 8 9}$ & $\mathbf{0 . 7 9 0}$ \\
\hline
\end{tabular}




\subsection{Approaches for Comparison}

The effectiveness of our model is evaluated by comparing with the state-of-the-art methods. The contrastive methods include traditional machine learning model e.g. SVM and the neural network methods as follows:

- SVM: is the basic classification model which uses n-gram and tf-idf features for rumor detection. We set it as the baseline in our experiment;

- SVM-UN: is the SVM model with uncertainty feature for rumor detection;

- ML-GRU [8]: uses deep recurrent neural networks to represent the semantics for rumor detection. This model is considered as a neural network baseline without accounting for uncertainty semantics;

- CSI [11]: is the state-of-the-art approach;

- NERUD: is our proposed model which exploits uncertainty attention mechanism to capture the uncertainty expression for rumor detection.

- NERUD*: NERUD without uncertainty identifier.

\subsection{Result and Discussion}

We firstly compare the effectiveness of NERUD with other approaches on two datasets shown in Tables 3 and 4, and then showcase attention mechanism.

NERUD can effectively detect the rumor on social media texts. On Ma's dataset, although NERUD does not always outperform the others, its performance is close to the best one. On CRC dataset, NERUD achieves the best F1 score. Note that in our experiments, all the neural networks for rumor detection are trained based on Ma's dataset, but only NERUD achieves comparable performance on CRC dataset. It is because that the event semantics are hardly maintained on a new dataset, while the uncertainty semantics will be consistent with the changes of topics.

Table 3. Performance on Ma's dataset.

\begin{tabular}{l|l|l|l}
\hline Method & Precision & Recall & F1 \\
\hline SVM & 0.673 & 0.746 & 0.707 \\
\hline SVM-UN & 0.703 & 0.766 & 0.733 \\
\hline ML-GRU & 0.871 & $\mathbf{0 . 9 5 8}$ & 0.913 \\
\hline CSI & - & - & $\mathbf{0 . 9 5 4}$ \\
\hline NERUD* & 0.898 & 0.932 & 0.914 \\
\hline NERUD & $\mathbf{0 . 9 1 3}$ & 0.941 & 0.926 \\
\hline
\end{tabular}

Uncertainty feature is useful for rumor detection. By integrating the uncertainty feature into rumor detection, the F1 scores are better than those basic versions without uncertainty ones. It confirms that uncertainty expressions help in detecting rumors in social media texts.

We also visualize rumor representation with different attentions generated by NERUD in a heatmap shown in Fig. 3. It is observed that NERUD highlights different 
Table 4. Performance on CRC dataset.

\begin{tabular}{l|l|l|l}
\hline Method & Precision & Recall & F1 \\
\hline SVM & 0.603 & 0.705 & 0.650 \\
\hline SVM-UN & 0.687 & 0.731 & 0.708 \\
\hline ML-GRU & 0.819 & 0.880 & 0.848 \\
\hline CSI & 0.831 & 0.906 & 0.867 \\
\hline NERUD* & 0.825 & 0.894 & 0.858 \\
\hline NERUD & $\mathbf{0 . 8 7 2}$ & $\mathbf{0 . 9 2 1}$ & $\mathbf{0 . 8 9 6}$ \\
\hline
\end{tabular}

types of words for rumor attention and uncertainty attention. For rumor attention, NERUD highlights topical words such as salt and nuclear. For uncertainty attention, NERUD highlights non-topic words, e.g., probably and could that express uncertainty semantics. Therefore, features learned for topical semantics and uncertainty semantics can thus benefit rumor detection.

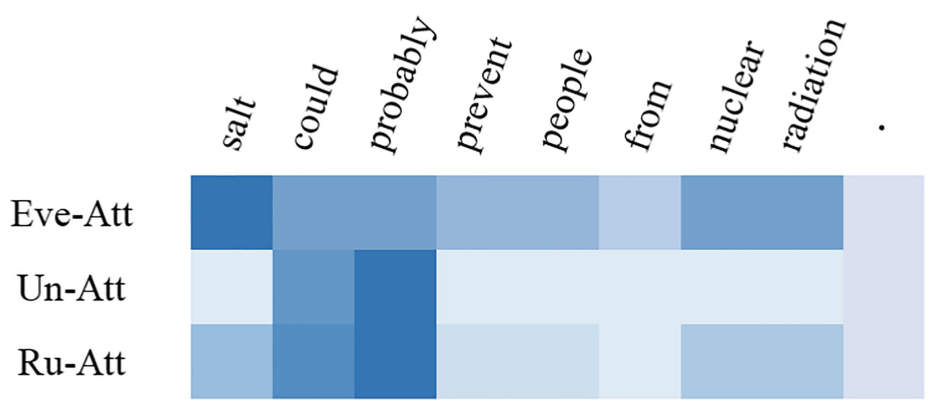

Fig. 3. The heatmap of the rumor attention(Ru Att), uncertainty attention(Un Att), and their combination generated by NERUD. Darker colors indicate higher weights.

\section{Related Works}

Traditionally, research on rumor detection has mainly focused on developing handcrafted features for machine learning algorithms [12]. [13] proposed a method for rumor detection on Twitter using cue words and tweets statistics. [17] applied two new types of features - client-based and location-based features - to rumor detection on Sina Weibo. Beyond this, user-based [6] and topic-based [15] features have also been explored. [4] demonstrated that there were structural differences in the propagation of rumors and non-rumors, and [14] and [9] experiment with using these propagation patterns extensively to improve detection.

More recently, deep learning models are explored for the task. Compared to traditional machine learning approaches, these deep learning models tended to rely less on sophisticated handcrafted features. [8] introduced a rumor detection model for microblogs based on recurrent networks. The input to their model was simple $t f$-idf features but it outperforms models leveraging handcrafted features. [18] showed that implicit 
linkages between conversation fragments improve detection accuracy. [7] presented a deep attention model that learns a hidden temporal representation for each sequential posts to represent the hypothesis. [11] integrated textual, user response, and source information into their neural models and achieved better performance.

\section{Conclusions}

In this paper, we present a neural rumor detection framework, namely NERUD. NERUD incorporates the uncertainty semantics into rumor representation through attention mechanism. Experiments were conducted on two Sina Weibo datasets, and the results showed that our NERUD outperformed state-of-the-art approaches on CRC dataset, and the uncertainty semantics is proven effective on rumor detection task.

Acknowledgement. This work is partially funded by the National Natural Science Foundation of China (61602326, U1636103, 61672361, and U1536207) and the Fundamental Research Fund for the Central Universities (3262019T29 and 3262019T54).

\section{References}

1. Allport, G.W., Postman, L.J.: The psychology of rumor. Russell\&Russell (1965)

2. Bahdanau, D., Cho, K., Bengio, Y.: Neural machine translation by jointly learning to align and translate. arXiv preprint arXiv:1409.0473 (2014)

3. Duchi, J., Hazan, E., Singer, Y.: Adaptive subgradient methods for online learning and stochastic optimization. J. Mach. Learn. Res. 12, 2121-2159 (2011)

4. Drien, F., Lada, A.A., Dean, E., Justin, C.: Rumor cascades. In: Proceedings of ICWSM (2014)

5. Li, B., Zhou, K., Gao, W., Han, X., Zhou, L.: Attention-based LSTM-CNNs for uncertainty identification on Chinese social media texts. In: Proceedings of ICSPAC (2017)

6. Liang, G., He, W., Chun, X., Chen, L., Zeng, J.: Rumor identification in microblogging systems based on users' behavior. IEEE Trans. Comput. Soc. Syst. 2(3), 99-108 (2015)

7. Long, Y., Lu, Q., Xiang, R., Li, M., Huang, C.-R.: Fake news detection through multiperspective speaker profiles. In: Proceedings of the Eighth International Joint Conference on Natural Language Processing (Volume 2: Short Papers), vol. 2 (2017)

8. Ma, J., et al.: Detecting rumors from microblogs with recurrent neural networks. In: Proceedings of IJCAI (2016)

9. Ma, J., Gao, W., Wong, K.-F.: Detect rumors in microblog posts using propagation structure via Kernel learning. In: Proceedings of the 55th Annual Meeting of the ACL (2017)

10. Mikolov, T., Sutskever, I., Chen, K., Corrado, G., Dean, J.: Distributed representations of words and phrases and their compositionality. In: Proceedings of NIPS 2013 (2013)

11. Natali, R., Seo, S., Liu, Y.: CSI: a hybrid deep model for fake news detection. In: Proceedings of the 2017 ACM on Conference on Information and Knowledge Management. ACM (2017)

12. Qazvinian, V., Rosengren, E., Radev, D.R., Mei, Q.: Rumor has it: identifying misinformation in microblogs. In: Proceedings of EMNLP, pp. 1589-1599 (2011) 
13. Takahashi, T., Nobuyuki, I.: Rumor detection on twitter. In: 2012 Joint 6th International Conference on Soft Computing and Intelligent Systems (SCIS) and 13th International Symposium on Advanced Intelligent Systems (ISIS). IEEE (2012)

14. Wu, K., Yang, S., Zhu, K.Q.: False rumors detection on sina weibo by propagation structures. In: 2015 IEEE 31st International Conference on Data Engineering, pp. 651-662 (2015)

15. Yang, F., Liu, Y., Yu, X., Yang, M.: Automatic detection of rumor on sina weibo. In: Proceedings of the ACM SIGKDD Workshop on Mining Data Semantics (2012)

16. Zubiaga, A., Aker, A., Bontcheva, K., Liakata, M., Procter, R.: Detection and resolution of rumours in social media: a survey. arXiv preprint arXiv:1704.00656 (2017)

17. Yang, F., Liu, Y., Yu, X., Yang, M.: Automatic detection of rumor on sina weibo. In: Proceedings of the ACM SIGKDD Workshop on Mining Data Semantics, p. 13. ACM (2012)

18. Sampson, J., Morstatter, F., Wu, L., Liu, H.: Leveraging the implicit structure within social media for emergent rumor detection. In: Proceedings of the 25th ACM International on Conference on Information and Knowledge Management, pp. 2377-2382. ACM (2016) 